\title{
Peptide vaccine with glucopyranosyl lipid A-stable oil-in-water emulsion for patients with resected melanoma
}

\author{
Eric P Grewal ${ }^{1}$, Courtney L Erskine ${ }^{2}$, Wendy K Nevala ${ }^{3}$, Jacob B Allred ${ }^{4}$, Carrie A Strand ${ }^{4}$, \\ Lisa A Kottschade ${ }^{1}$, Robert R McWilliams ${ }^{1}$, Roxana S Dronca ${ }^{5}$, Adam J Yakovich ${ }^{6}$, Svetomir \\ N Markovic ${ }^{1} \&$ Matthew S Block*,1 (iD \\ ${ }^{1}$ Mayo Clinic Rochester, Division of Medical Oncology, 200 First Street SW, Rochester, MN 55905, USA \\ ${ }^{2}$ Department of Immunology, 200 First Street SW, Rochester, MN 55905, USA \\ ${ }^{3}$ Division of Oncology Research, 200 First Street SW, Rochester, MN 55905, USA \\ ${ }^{4}$ Department of Biostatistics \& Informatics, 200 First Street SW, Rochester, MN 55905, USA \\ ${ }^{5}$ Department of Hematology/Oncology, Mayo Clinic Jacksonville, 4500 San Pablo Road, Jacksonville, FL 32224, USA \\ ${ }^{6}$ Immune Design, Inc., 1616 Eastlake Ave E \#310, Seattle, WA 98102, USA \\ *Author for correspondence: Tel.: +1 507293 0462; Block.matthew@mayo.edu
}

\begin{abstract}
Aim: We tested the safety and immunogenicity of a novel vaccine in patients with resected high-risk melanoma. Patients \& methods: HLA-A2-positive patients with resected Stage II-IV melanoma were randomized to receive up to three vaccinations of melanoma-associated peptide (MART-1a) combined with a stable oil-in-water emulsion (SE) either with the Toll-like receptor 4 agonist glucopyranosyl lipid A (GLASE-Schedule 1) or alone (SE-Schedule 2). Safety and immunogenicity of the vaccines were monitored. Results: A total of 23 patients were registered. No treatment-related grade 3 or higher adverse events were observed. Increases in MART-1a-specific T cells were seen in 70 and $63 \%$ of Schedule 1 and Schedule 2 patients, respectively. Conclusion: Both vaccine schedules were well-tolerated and resulted in an increase in MART-1a-specific T cells. Clinical Trial registration: NCT02320305 (ClinicalTrials.gov).
\end{abstract}

First draft submitted: 31 March 2020; Accepted for publication: 16 July 2020; Published online: 5 August 2020

Keywords: glucopyranosyl lipid A • immunotherapy • MART-1 • melanoma $\bullet$ peptide $\bullet$ Phase I $\bullet$ T cell $\bullet$ vaccine

Despite landmark advances in the field of cancer immunotherapy, cutaneous melanoma remains a deadly disease for patients with advanced or metastatic disease. Patients often undergo surgical excision, a therapy that can be curative, but is frequently associated with recurrence in Stage III and IV disease [1]. Even by combining surgical resection with the use of adjuvant checkpoint inhibitor immunotherapies, nearly $30 \%$ of patients with resected advanced melanoma develop recurrence [2]. Novel therapies that can enhance the antitumor immune response and provide a durable survival benefit for patients are direly needed.

One promising strategy for treating advanced stage melanoma is the use of therapeutic vaccination. It has long been recognized that tumor cells express antigens that can be recognized by the host immune system [3]. Furthermore, multiple clinical studies have demonstrated the ability to activate cytotoxic T lymphocytes (CTLs) against tumor-relevant antigens $[4,5]$. In particular, MART-1 is recognized by host tumor-infiltrating lymphocytes from patients with metastatic melanoma and has been proposed as a target for therapeutic vaccination [6]. In the past, our group has demonstrated the ability to induce MART-1-specific immune responses by using MART-1a (ELAGIGILTV), a heteroclitic peptide that has been shown to activate patient CD8 T cells in vivo [7]. However, due to immune tolerance, the use of peptides without adjuvants has not been sufficient for inducing durable immune responses in cancer patients [8].

The majority of anticancer peptide vaccines being evaluated today utilize adjuvants that ligate host patternrecognition receptors to induce pro-inflammatory innate immune signaling [9]. This innate immune activation is key for the upregulation of antigen presentation and costimulatory molecules on dendritic cells, which have been shown to induce antigen-specific CD8 T cells in the context of tumor vaccination [10]. Many of these adjuvants, 
such as polyinosinic:polycytidylic acid, monophosphoryl lipid A, resiquimod and $\mathrm{CpG}$ oligodeoxynucleotides, target Toll-like receptors (TLRs) [11]. In particular, glucopyranosyl lipid A (GLA) combined with stable oil-in-water emulsion (SE) is a potent TLR4 agonist that has been shown preclinically to enhance antigen-specific immune responses in the context of $\mathrm{T}_{\mathrm{H}} 1$ immunity without any major safety concerns [12]. GLA-SE has also been used in an experimental tuberculosis vaccine, where its use was associated with a strong, polyfunctional $\mathrm{T}_{\mathrm{H}} 1$ response [13].

Since $\mathrm{T}_{\mathrm{H}} 1$ immunity is thought to be the optimal context for development of an antitumor CTL immune response [14], we have conducted a clinical trial to test the safety and immunogenicity of MART-1a peptide combined with GLA-SE or SE alone in patients with resected stage II-IV melanoma, with the primary aim of assessing vaccine-induced patient CD8 $\mathrm{T}$ cell responses against MART-1. The secondary aim of this study is to establish the safety of vaccination with GLA-SE in cancer patients, with monitoring of any serious side effects or adverse events. Patient parameters, such as plasma cytokine changes or shifts in key immune cell populations, are also monitored to understand the immunological effects of vaccination with this regimen.

\section{Patients \& methods}

\section{Patient characteristics}

This study included patients 18 years of age or older with histologically confirmed stage II-IV melanoma that had been completely resected or treated with ablative therapy prior to trial commencement, with no current evidence of disease as assessed by chest x-ray (acceptable for stage II patients only), computed tomography (CT), MRI or positron emission tomography/CT. Patients were eligible if they were HLA-A2-positive, had an Eastern Cooperative Oncology Group score of $0-2$, had a life expectancy of at least 12 weeks and demonstrated positive MART-1 expression via central pathology review. Additional inclusion criteria included an absolute neutrophil count greater than $1500 / \mu \mathrm{l}$, hemoglobin greater than $10 \mathrm{~g} / \mathrm{dl}$, platelet count greater than $150,000 / \mu \mathrm{l}$ and an aspartate aminotransferase and alkaline phosphatase of less than three-times the upper limit of normal. Patients must have been capable of providing informed consent, willing to return to Mayo Clinic for follow-up and able to provide blood samples for study evaluation. All women of childbearing potential were also required to screen negatively on urine pregnancy test within 7 days prior to registration. Patients were excluded if they had an ongoing infection, any known immune deficiencies (including HIV) or systemic autoimmune diseases, received prior therapy (chemotherapy, radiation therapy, biologic or immunologic therapy) or immunosuppressive medications within 4 weeks prior to registration, or possessed any known allergies to any vaccine or adjuvant components (including egg). Additional exclusion criteria included pregnancy or the potential to become pregnant, breastfeeding, active brain metastases, other active malignancies or eligibility for any known standard therapy. The protocol was approved by the Mayo Clinic Institutional Review Board and all patients underwent written informed consent prior to registration and study participation. The research was conducted according to the principles of the Declaration of Helsinki. The study was registered at ClinicalTrials.gov as NCT02320305.

\section{Vaccine preparation}

MART-1a peptide (ELAGIGILTV) was synthesized by PolyPeptide Laboratories (CA, USA). For patients treated on Schedule 1, equal volumes of reconstituted MART-1a peptide and GLA-SE, a TLR4 agonist in a stable oil-in-water emulsion (Immune Design, WA, USA) were mixed to produce a vaccine of $0.5 \mathrm{mg} / \mathrm{ml}$ MART-1a with $5 \mathrm{mcg} / \mathrm{ml}$ GLA-SE emulsion. For patients treated on Schedule 2, equal volumes of reconstituted MART-1a peptide and SE, the stable oil-in-water emulsion alone (Immune Design), were mixed to produce a vaccine of $0.5 \mathrm{mg} / \mathrm{ml}$ MART-1a in SE. For both schedules, a total vaccine volume of $1 \mathrm{ml}$ was used.

\section{Vaccine administration}

Prior to registration and after each cycle of treatment, each patient received a physical examination, radiologic disease evaluation, toxicity assessment using Common Terminology Criteria for Adverse Events version 4.0 and analysis of hematologic and chemical laboratory values. Patients who did not meet criteria for registration were excluded from the study. Upon registration, patients were randomized to one of two treatment schedules (Figure 1). Doses were not escalated for patients in either schedule. Vaccinations were administered as single intramuscular injections into a region of undisturbed axillary or inguinal lymph nodes. For subsequent vaccination cycles, a lymph node drainage site that had not previously been used for that patient was targeted for administration, if available. Otherwise, the lymph node drainage site targeted in the first vaccination cycle was used again. Vaccines were administered at the beginning of 21-day cycles, with each patient receiving up to three cycles of treatment 


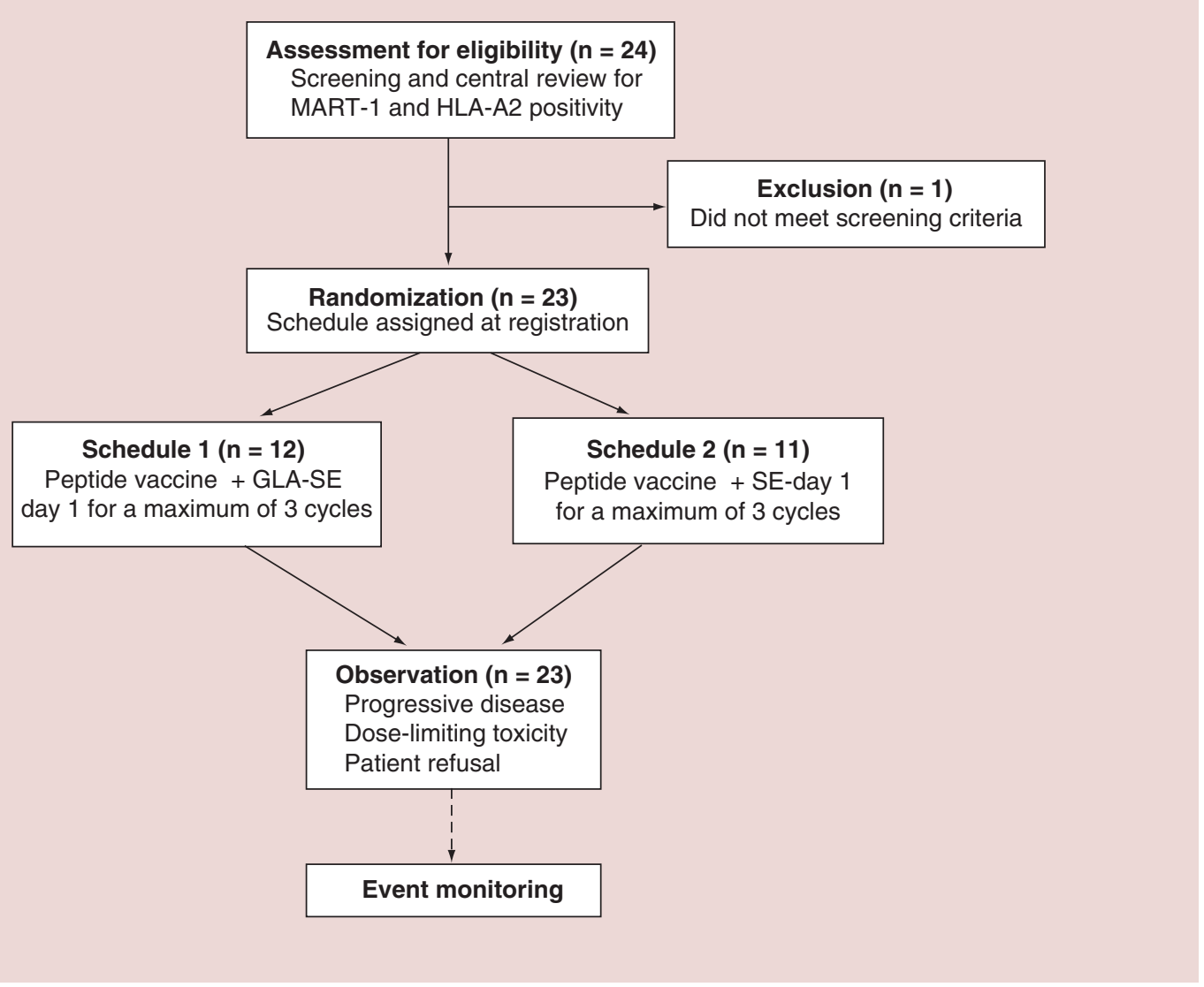

Figure 1. Study schematic.

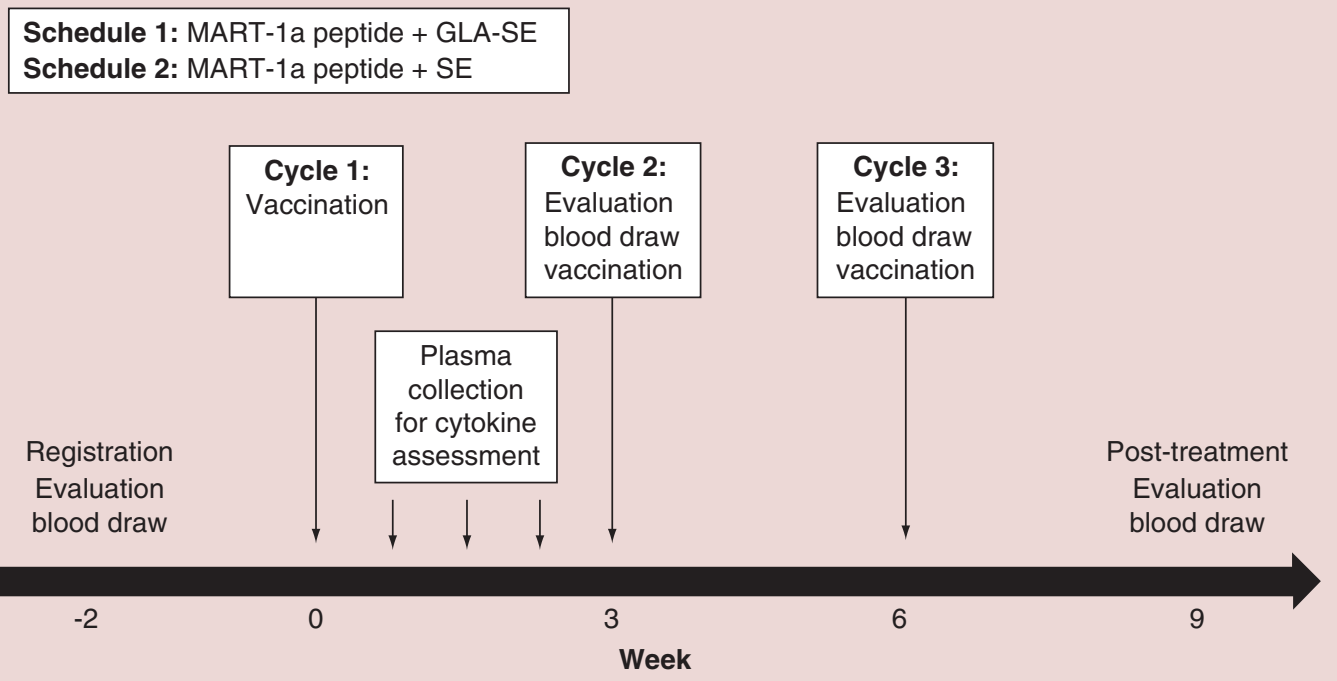

Figure 2. Vaccine administration.

(Figure 2). Dose-limiting toxicities were defined as greater than or equal to grade 2 autoimmune disorders, greater than or equal to grade 2 hypersensitivity reactions, greater than or equal to grade 3 injection site reactions, greater 
than or equal to grade 3 myalgia/arthralgia or bone pain, an absolute neutrophil count of less than 1000 or platelet count of less than 50,000, or any other greater than or equal to grade 3 nonhematologic toxicity that was definitely, probably, or possibly related to the study treatment. In addition to each postcycle evaluation, patients underwent disease evaluation via physical examination, laboratory testing and computed tomography or positron emission tomography/CT imaging every 6 months for patients with Stage II/IIIA disease and every 3 months for patients with Stage IIIB/IIIC/IV disease, for up to 24 months of follow-up. This study was initiated in January 2015, with enrollment proceeding until January 2016 and preliminary study completion in April 2019.

\section{Antigen-specific immune responses}

Peripheral blood mononuclear cells (PBMCs) were collected prior to each cycle of vaccine treatment and 3 weeks after the third vaccine treatment, as well as every 6 months during the 2-year observation period. Quantification of MART-1a-specific CD8 T cells was performed using the IFN- $\gamma$ enzyme-linked immunosorbent spot (ELISpot) assay. Briefly, thawed PBMCs from patient samples were plated at a concentration of $2.5 \times 10^{5}$ cells per well and stimulated for 24 hours with culture medium alone or $10 \mathrm{mg} / \mathrm{ml}$ of peptide. Four test peptide groups were used: MART-1a; MART-1; cytomegalovirus (C), Epstein-Barr Virus (E) and influenza virus (F) (CEF) positive control; and HIV Gag negative control. ELISpot plates (Millipore, MA, USA) were prepared by coating with $10 \mu \mathrm{g} / \mathrm{ml}$ of IFN- $\gamma$ capture antibody (MabTech USA, OH, USA), followed by incubation for $24 \mathrm{~h}$. Subsequently, ELISpot plates were rinsed with phosphate-buffered saline (PBS) and underwent blocking using standard cell culture medium for $2 \mathrm{~h}$. To prepare for assay execution, patient PBMCs that had undergone stimulation were transferred to the prepared ELISpot plates for $24 \mathrm{~h}$ of incubation at $37^{\circ} \mathrm{C}$. Afterward, the plates were washed using $0.05 \%$ Tween-20 in PBS. Next, $2 \mu \mathrm{g} / \mathrm{ml}$ of biotinylated IFN- $\gamma$ secondary antibody (MabTech USA) was added for detection, followed by incubation for $2 \mathrm{~h}$ at $37^{\circ} \mathrm{C}$. The plates were again rinsed with $0.05 \%$ Tween-20 in PBS before addition of streptavidin-horseradish peroxidase (BD Biosciences, CA, USA), which was diluted at $1 \mu \mathrm{l}$ per $1 \mathrm{ml}$ of PBS containing $10 \%$ fetal bovine serum. After incubation for one additional hour at room temperature, plates were first rinsed with $0.05 \%$ Tween-20 in PBS before a final rinse using pure PBS. To develop the plates, 3-amino-9-ethyl-carbazole peroxidase substrate (Sigma-Aldrich, MO, USA) was added to each well and allowed to react. After quenching the reaction with water, plates were allowed to dry overnight before reading on an iSpot EliSpot reader (AID GmbH, Strasberg, Germany), which quantitates spots based on the number of cells per well that were stimulated to secrete IFN- $\gamma$. To reduce variability, samples were plated in triplicate wells and the final number of spots per sample was calculated by averaging the sum of each set of three wells. To reduce background noise and obtain the true number of antigen-specific cells per well, the number of spots obtained using medium alone was subtracted from the number of spots produced by each sample. In addition to using media as a negative control, phytohemagglutinin was employed as a positive control for T cell IFN- $\gamma$ production. ELISpot plates were kept after automated counting and were visually compared with the automated counts. Manual and automated counts were similar and so the ELISpot plates were discarded and are no longer available.

\section{Plasma cytokine assessment}

Plasma samples were collected from each patient between days 2-3, 6-8 and 12-15 of Cycle 1 and analyzed using the Bio-Plex Pro ${ }^{T M}$ Human Cytokine Assay (Bio-Rad, CA, USA). This assay enabled quantitation of soluble factors including CCL7, CCL10, CXCL1, CXCL10, EGF, FGF-2, FLT-3L, G-CSF, GM-CSF, IFN- $\alpha 2$, IFN- $\gamma$, IL-1 $\alpha$, IL-1 $\beta$, IL-1RA, IL-4, IL-6, IL-7, IL-8, IL-10, IL-12p40, IL-12p70, MCP-1, MIP-1 $\alpha$, MIP-1 $\beta$, sCD40L, TGF- $\alpha$ and TNF- $\alpha$. Assay plates were developed according to manufacturer instructions and were detected using a Luminex ${ }^{\circledR}$ plate reader (Luminex Corporation, TX, USA). To obtain protein concentrations, a standard curve was generated using the Bio-Plex Manager Software (Bio-Rad). For cytokines which displayed concentrations above (or below) the limit of assay detection, the value for the highest (or lowest) detectable concentration was substituted. Factors for which there was at least a doubling or halving in post-vaccination concentration for at least two patients per schedule were displayed.

\section{Immunophenotyping}

PBMCs collected from each patient at baseline and after each cycle were assessed via flow cytometry using fluorescein isothiocyanate-conjugated and R-phycoerythrin-conjugated antibodies to CD3, CD4, CD11c, CD14, CD16, CD19, CD33, CD62L, CD69, CD123, CD294, HLA-DR and TIM-3 (Supplementary Table 1). Data were processed using CellQuest software (BD Biosciences). Samples were assessed for the ratio of Th1 to Th2 cells 
Table 1. Patient characteristics.

\begin{tabular}{|c|c|c|}
\hline & Schedule 1: GLA-SE & Schedule 2: SE \\
\hline \multicolumn{3}{|l|}{ Age } \\
\hline$-N$ & 12 & 11 \\
\hline - Median & 56.0 & 48.0 \\
\hline - Range & $30.0,78.0$ & $28.0,82.0$ \\
\hline \multicolumn{3}{|l|}{ Sex, $n(\%)$} \\
\hline - Male & $7(58.3 \%)$ & $6(54.5 \%)$ \\
\hline \multicolumn{3}{|l|}{ Stage, n (\%) } \\
\hline-11 & $1(8.3 \%)$ & $0(0.0 \%)$ \\
\hline$-I I I$ & $7(58.3 \%)$ & $8(72.7 \%)$ \\
\hline$-I V$ & $4(33.3 \%)$ & $3(27.3 \%)$ \\
\hline - Lower extremity & $3(25.0 \%)$ & $3(27.3 \%)$ \\
\hline - Trunk & $2(16.7 \%)$ & $3(27.3 \%)$ \\
\hline - Unknown & $1(8.3 \%)$ & $2(18.2 \%)$ \\
\hline
\end{tabular}

$\left(\mathrm{CD} 4^{+}\right.$TIM-3 ${ }^{+}$to $\left.\mathrm{CD} 4{ }^{+} \mathrm{CD} 294^{+}\right)$using a gating strategy that has been described previously $[15,16]$. Additionally, CD69, which is expressed in early activation, was compared with CD62L, a marker of naive and central memory $\mathrm{T}$ cells, to capture the ratio of activated/central memory to naive $\mathrm{T}$ cells $\left(\mathrm{CD} 3^{+} \mathrm{CD}^{+} 9^{+}\right.$to $\left.\mathrm{CD}^{+} \mathrm{CD}^{+} \mathrm{L}^{+}\right)[17,18]$. The ratio of DC1 to DC2 cells (CD3- CD14- CD16- CD19- HLA-DR ${ }^{+}$CD11 ${ }^{+}$to CD3- CD14- CD16- CD19$\left.\mathrm{HLA}_{-} \mathrm{DR}^{+} \mathrm{CD}^{2} 2^{-}\right)$were also assessed. Fluorescence gates were established using unstained negative controls and the total number of events (i.e., cells) staining positive in each gate were collected. Cell ratios were obtained by calculating the quotient from the proportion of positively stained cells in each population being compared. Raw flow cytometry data were kept after analysis for 1 year but are no longer available at the time of this manuscript.

\section{Statistical considerations}

The primary end point of this investigative study was immune response as assessed by ELISpot quantification of MART-1a-specific CD8 T cells. A positive patient response was defined as a postvaccination number of antigenspecific $T$ cells that more than doubled from the prevaccination value to the maximal postcycle value, with a change greater than double the sum of the standard deviation between the prevaccination and maximal postvaccination value. Additionally, the criteria for establishing positive response in each vaccine schedule was determined to be at least six positive patient responses out of ten, in order for the lower bound of a binomial $95 \%$ CI to be $25 \%$ at minimum. The secondary end point of this trial was assessment of the grades and frequencies of any adverse events. Additional correlative analyses, including changes in aggregate plasma cytokine concentrations and key immune cell population frequencies, were performed to understand biological changes induced by vaccination. Descriptive statistics were used to summarize these data and analyses were performed using Prism (Version 8; GraphPad Software, CA, USA).

\section{Results}

\section{Demographics}

A total of 24 patients with resected melanoma were enrolled in the trial between February 2015 and January 2016, of whom 23 met eligibility requirements for registration (Figure 1). The majority of patients enrolled in this trial (65.2\%) had Stage III melanoma, while 30.4\% had Stage IV disease and one patient (4.3\%) had Stage II disease. A total of 12 patients were randomized to Schedule 1 and 11 patients were randomized to Schedule 2 (Figure 2). Patient characteristics are shown in Table 1. 


\begin{tabular}{|c|c|c|c|c|c|c|c|c|}
\hline \multirow[t]{3}{*}{ Event Type, n (\%) } & \multicolumn{4}{|c|}{ Schedule 1: GLA-SE } & \multicolumn{4}{|c|}{ Schedule 2: SE } \\
\hline & \multicolumn{4}{|c|}{ Grade } & \multicolumn{4}{|c|}{ Grade } \\
\hline & 1 & 2 & 3 & 4 & 1 & 2 & 3 & 4 \\
\hline Fatigue & $5(41.7 \%)$ & $1(8.3 \%)$ & - & - & $5(45.5 \%)$ & - & - & - \\
\hline Injection site reaction & $6(50 \%)$ & $1(8.3 \%)$ & - & - & $3(27.3 \%)$ & - & - & - \\
\hline Arthralgia & $3(25 \%)$ & $1(8.3 \%)$ & - & - & $1(9.1 \%)$ & - & - & - \\
\hline Platelet count decreased & $3(25 \%)$ & & - & - & $1(9.1 \%)$ & - & - & - \\
\hline Myalgia & $1(8.3 \%)$ & $1(8.3 \%)$ & - & - & $1(9.1 \%)$ & - & - & - \\
\hline Bone pain & $1(8.3 \%)$ & - & - & - & - & - & - & - \\
\hline $\begin{array}{l}\text { Neoplasms benign, malignant and } \\
\text { unspecified (including cysts and polyps) }\end{array}$ & - & - & - & - & - & - & $1(9.1 \%)$ & - \\
\hline
\end{tabular}

\section{Adverse events}

Patients were assessed for adverse events according to Common Terminology Criteria for Adverse Events Version 4 (Table 2). Overall, vaccination in both schedules was well-tolerated and not associated with any significant greater than or equal to grade 3 toxicity. One patient developed a grade 3 neoplasm: an early stage papillary thyroid cancer. Grade 2 adverse events were only observed in patients on Schedule 1; these included fatigue, injection site reaction, arthralgia and myalgia. The most common grade 1 adverse events in both schedules were fatigue and injection site reactions.

\section{Immunogenicity}

The frequency of IFN- $\gamma$-producing T cells specific for MART-1a, MART-1 and control peptides was assessed before and after treatment. In both schedules, there was a significant increase in the mean frequency of MART-1a-specific $\mathrm{T}$ cells without any significant increase in reactivity against the negative control Gag peptide, demonstrating the capability of vaccination with either GLA-SE or SE-containing peptide vaccine regimens to induce an antigenspecific immune response (Figure 3A). There was a trend toward increased reactivity toward the CEF peptide pool that did not reach statistical significance; $70.0 \%$ of patients vaccinated with GLA-SE and $63.6 \%$ of patients vaccinated with SE had positive immune responses to vaccination with MART-1a (Figure 3B). There was also an increase in the frequency of T cells specific for MART-1, with $40.0 \%$ of patients vaccinated with GLA-SE and $54.5 \%$ of patients vaccinated with SE exhibiting positive immune responses against MART-1. This provides evidence that vaccination with MART-1a can induce cross-reactivity against the tumor-relevant antigen MART-1.

\section{Plasma cytokine changes}

Systemic cytokine change is a key means to probe for vaccine-induced modulation of the immune response. To assess these changes, plasma samples were taken from patients at baseline and at three subsequent timepoints in Cycle 1 (days 2-3, 6-8 and 12-15). The maximal log-twofold changes in plasma cytokine concentration from baseline were calculated for each patient and displayed for each vaccine schedule (Figure 4A). Mean patient changes in acute inflammatory proteins were observed throughout Cycle 1, with elevations in IL-1 $\beta$, IL-1RA and IL-6 being most dramatically sustained in the SE arm compared with the GLA-SE arm (Figure 4B). Additional cytokines including TNF- $\alpha$, IL-4 and IL-10 demonstrated modest changes from baseline, with a greater elevation also in the SE arm compared with the GLA-SE arm. Factors that were similarly elevated in both treatment groups included MCP-1, EGF, TGF- $\alpha$ and sCD40L (Figure 4C). Interestingly, the chemokines MIP- $1 \alpha$ and MIP- $1 \beta$ demonstrated a progressive decrease in patients vaccinated with GLA-SE compared with SE.

\section{Cell populations}

The ratios of key immune cell populations were assessed at baseline and at the end of each treatment cycle. At baseline, the $T_{H} 1 / T_{H} 2$ ratio was noted to be higher in the SE group, but both groups exhibited a progressive increase in the $T_{H} 1 / T_{H} 2$ ratio (Figure $5 \mathrm{~A}$ ). In contrast, while the ratio of activated to naive/central memory $T$ cells was initially higher in the SE arm, post-Cycle 3 values were similar for both groups (Figure 5B). Finally, the $\mathrm{DC} 1 / \mathrm{DC} 2$ ratio did not exhibit a trend of change and ratio remained similar for both arms throughout the trial (Figure 5C). 

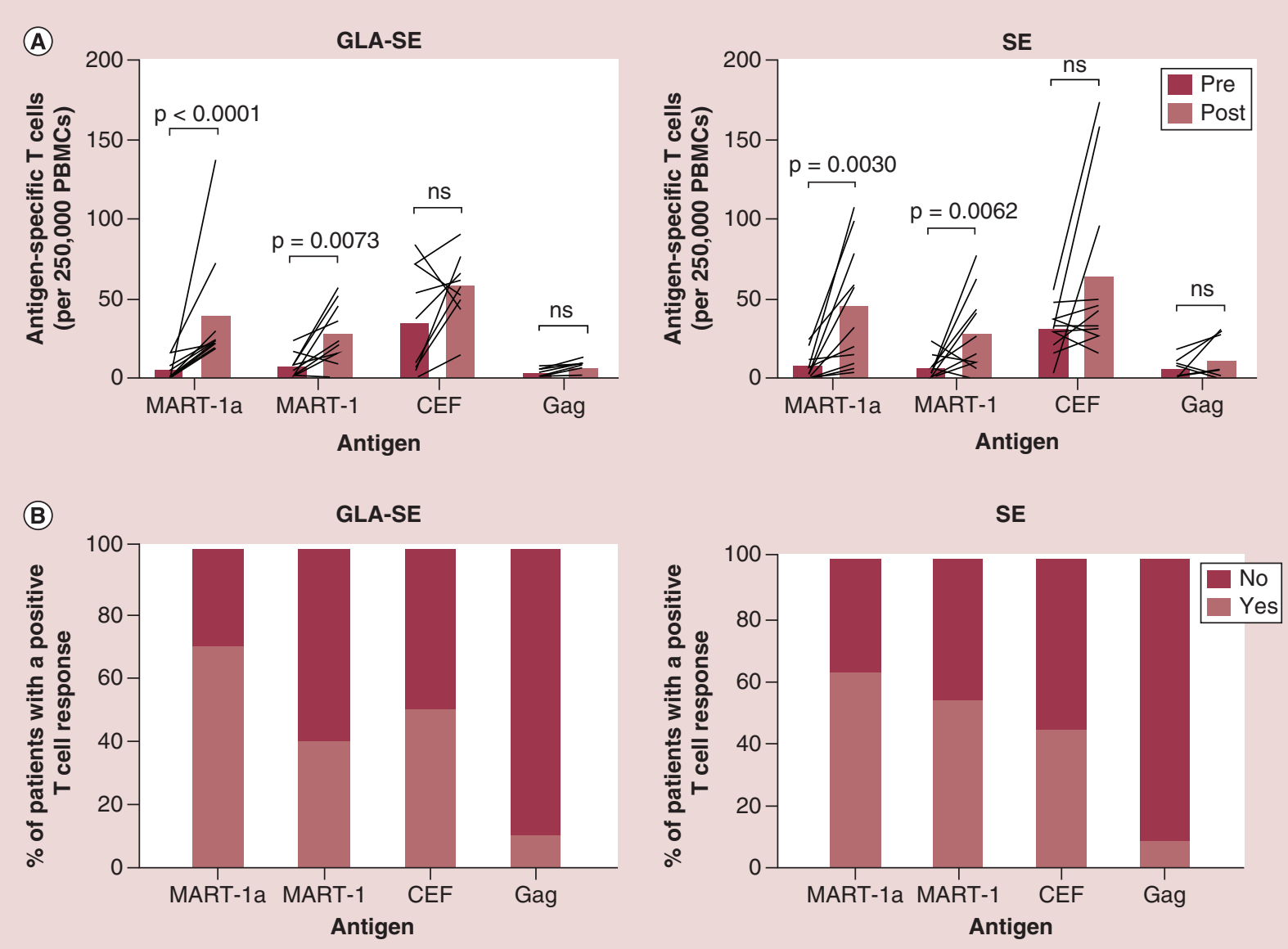

Figure 3. Assessment of antigen-specific immune responses to vaccination. (A) Mean prevaccination and maximal postvaccination frequency of antigen-specific T cells to vaccine antigen (MART-1a), MART-1, CMV, EBV and influenza (CEF) control peptide pool and irrelevant peptide control (HIV Gag) detected via ELISPOT for GLA-SE $(n=11)$ and SE $(n=11)$ treatment arms. Each line denotes a single patient. Significance testing was performed using the Wilcoxon rank-sum test $(p<0.05$ defined as significant. (B) Percentage of patients responding to each antigen.

ELISpot: Enzyme-linked immunosorbent spot; GLA-SE: Glucopyranosyl lipid A-stable oil-in-water emulsion; ns: Not significant; SE: Stable oil-in-water emulsion.

\section{Patient outcomes}

Of the 23 total patients enrolled in this trial, recurrence occurred in eight patients during 24 months of followup $-50.0 \%(6 / 12)$ of patients vaccinated on Schedule 1 and 18.2\% (2/11) of patients vaccinated on Schedule 2. Risk of recurrence across both schedules was associated with melanoma stage, with $75.0 \%$ of patients with Stage II-III melanoma remaining recurrence-free at a maximum follow-up of 24 months, but only $42.9 \%$ of patients with resected Stage IV melanoma remaining recurrence-free. Of the patients who remained recurrence-free across both schedules, $57.1 \%$ demonstrated positive postvaccination immune responses against MART-1a. Additionally, patients vaccinated on Schedule 1 who recurred on study had increases in select plasma cytokine levels relative to patients who remained recurrence-free during follow-up (Figure 6).

\section{Discussion}

Peptide vaccination remains a promising immunotherapeutic strategy for the treatment of melanoma. Although no peptide vaccines for melanoma have been licensed to date, strategies that employ novel combinations of adjuvants and antigens have the potential to enhance the antitumor immune response and produce a successful vaccine [19]. In this study, we aimed to assess the safety and immunogenicity of a MART-1a peptide vaccine with the immune adjuvant GLA-SE, the combination of a TLR4 agonist and a stable oil-in-water emulsion, compared with SE alone 

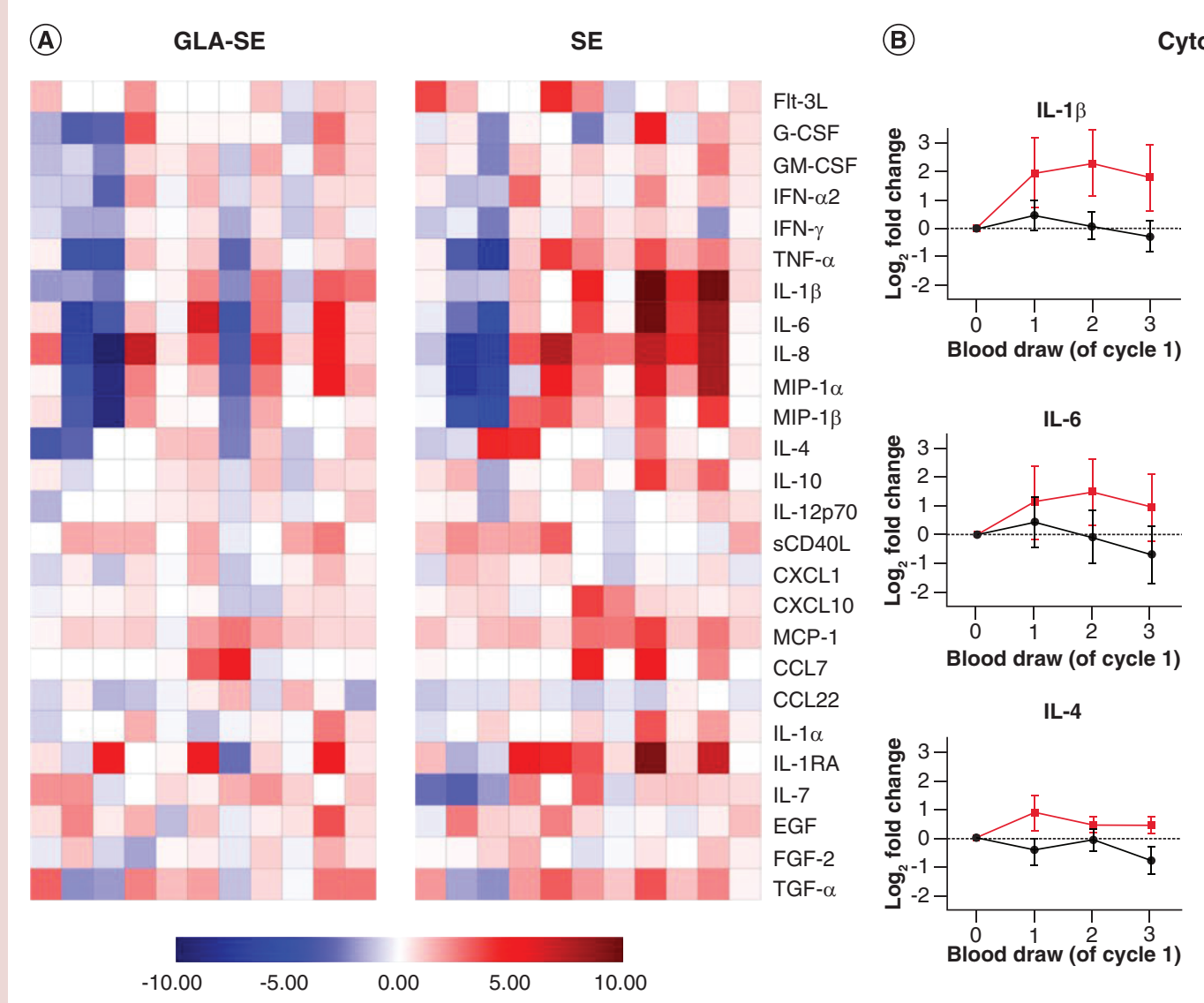

$-\mathrm{GLA}-\mathrm{SE}$
$-\mathrm{SE}$
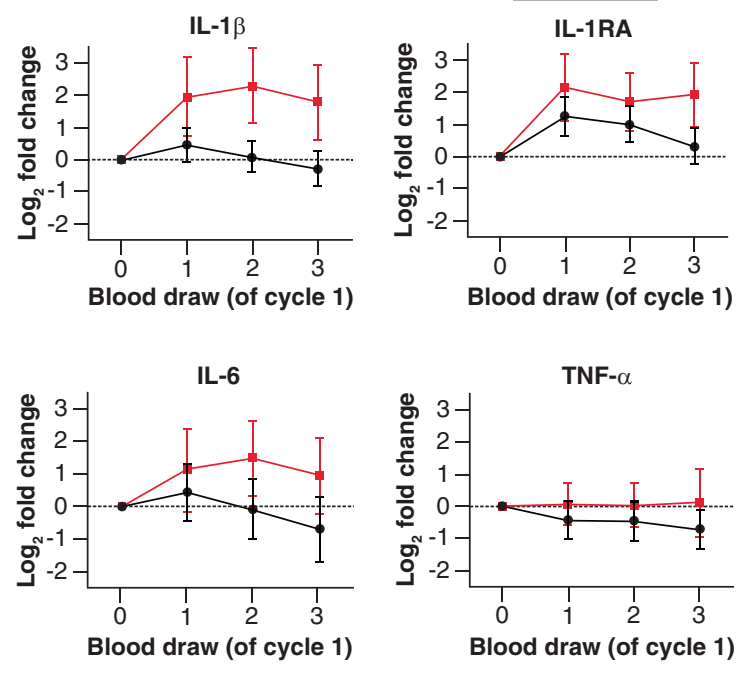

Blood draw (of cycle 1)

(C)

\section{Chemokines and growth factors}
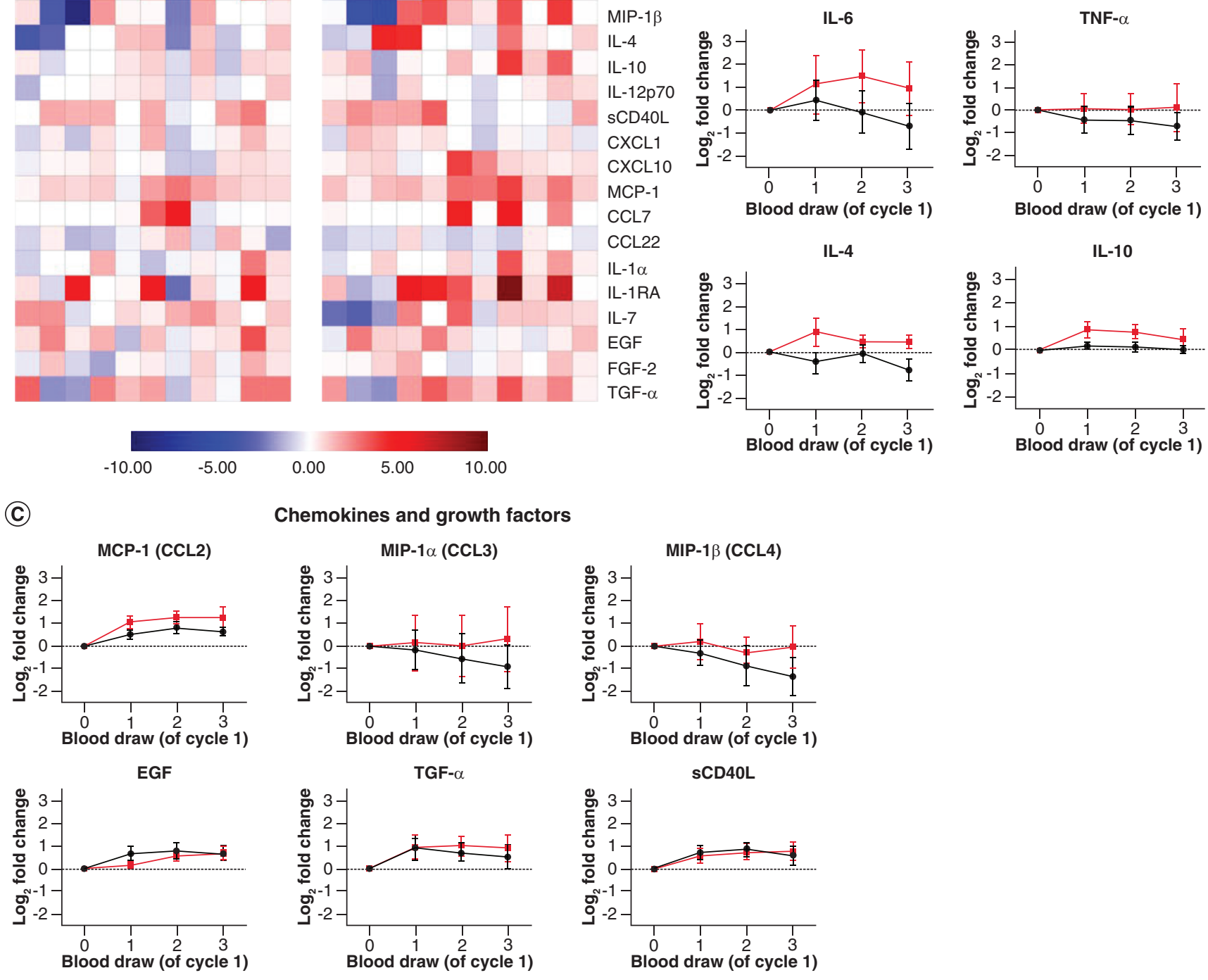

IL-10

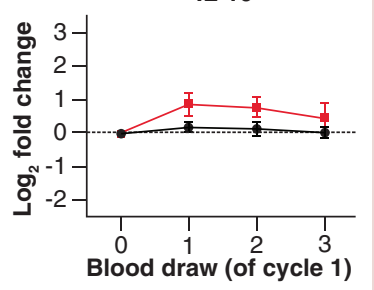

Figure 4. Analysis of plasma cytokine, chemokine and growth factor concentrations before and after vaccination. (A) Heatmap displaying the maximal post-vaccination change in soluble factor concentration (log-twofold-change from prevaccination level). Each column represents one patient. x-axis signifies blood draws correlating with baseline and days 2-3, 6-8 and 12-15 of Cycle 1, respectively. (B) Mean log-twofold change in selected cytokine and (C) chemokine/growth factor concentrations per treatment arm across Cycle 1. Error bars denote standard error of mean of values for each patient. 

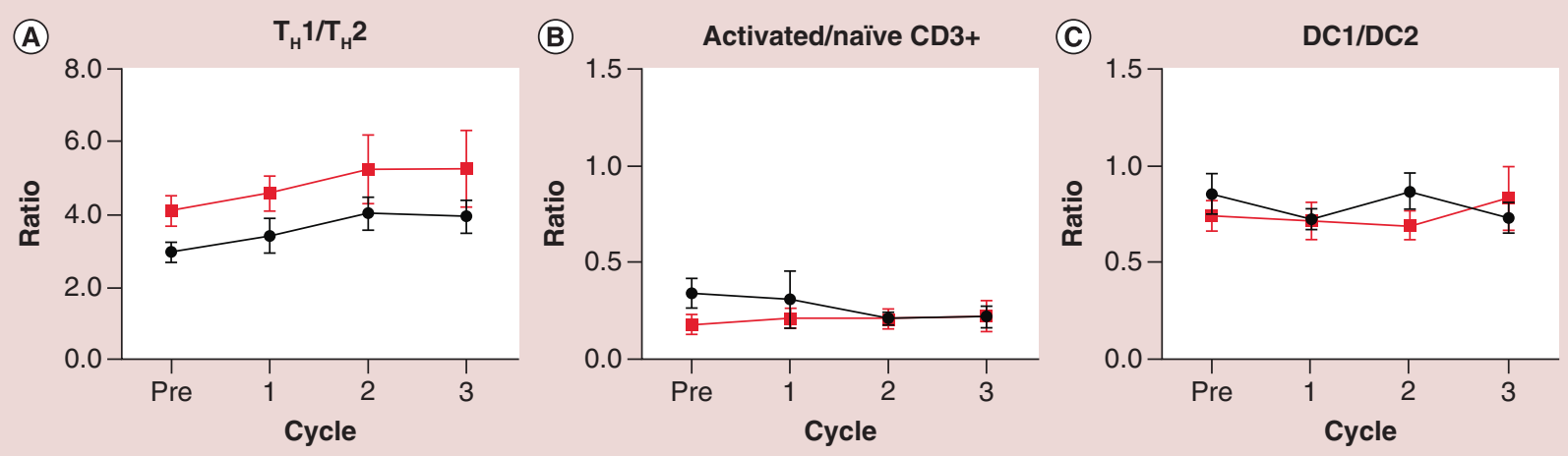

- GLA-SE

Figure 5. Changes in circulating immune cell populations throughout vaccine administration schedule. Ratios of FACS-sorted cell populations percentages were assessed at the end of each treatment cycle and averaged per treatment arm for (A) $T_{H} 1$ cells and $T_{H} 2$ cells; (B) activated/central memory $\left(\mathrm{CD} 69^{+}\right) \mathrm{CD}^{+} \mathrm{T}$ cells and naive $\left(\mathrm{CD} 2 \mathrm{~L}^{+}\right) \mathrm{CD}^{+} \mathrm{T}$ cells; and $(\mathrm{C}) \mathrm{DC} 1$ and DC2. Error bars denote standard error of mean of ratios for each patient.

DC1: Type 1 dendritic cell; DC2: Type 2 dendritic cell; $T_{H} 1$ : T helper $1 ; T_{H} 2:$ T helper 2.
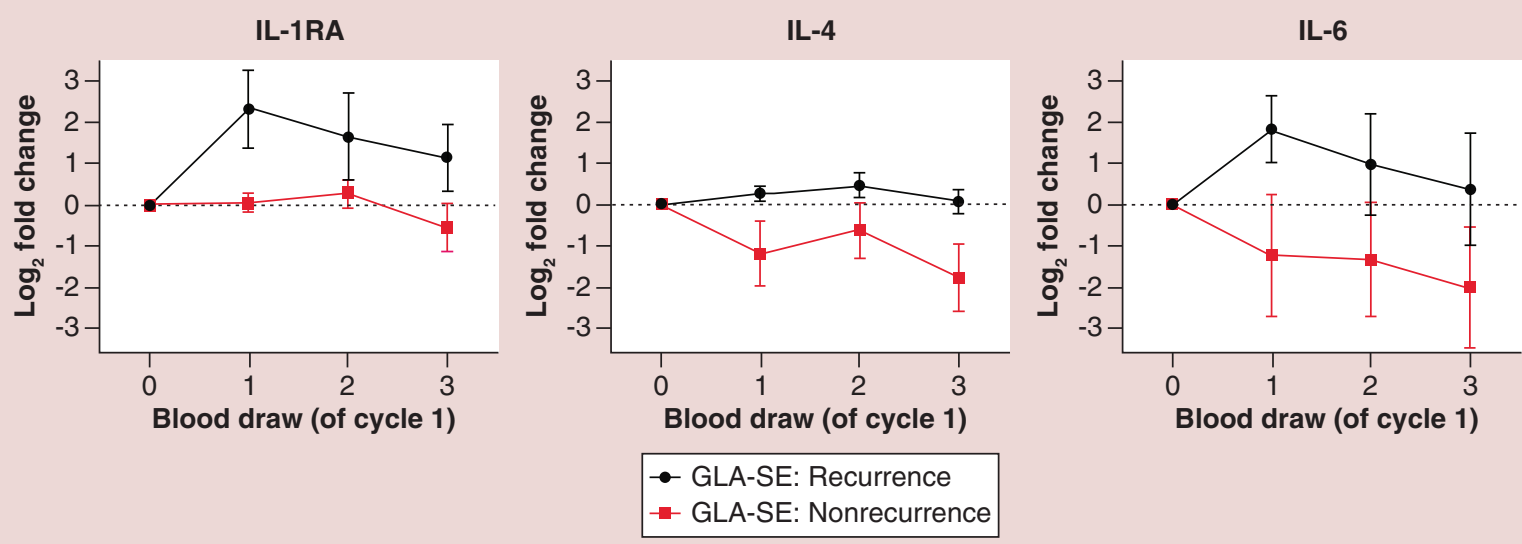

Figure 6. Plasma cytokine changes in patients with and without recurrences on Schedule 1. Data are shown as mean log-twofold change in selected cytokine. Error bars denote standard error of mean of values for each patient. x-axis signifies blood draws correlating with baseline and days $2-3,6-8$ and $12-15$ of Cycle 1 , respectively.

in patients with resected melanoma. Of note, the trial was a first-in-human pilot-sized clinical trial; this prevents a statistically powered comparison between the two groups and is a limitation to the study.

Vaccination with either formulation was demonstrated to be safe, with the majority of adverse events being grade 2 or lower fatigue and injection site reactions. A higher incidence of these adverse events was noted in Schedule 1 , which may be indicative of a greater innate inflammatory response when GLA and SE were delivered together compared with SE alone. The synergistic effect of GLA used in combination with SE has been noted previously and may be due to formation of a vaccine depot [20].

Both vaccine formulations demonstrated favorable immunogenicity by inducing IFN- $\gamma$-producing immune responses against the heteroclitic vaccine peptide MART-1a as well as its naturally-occurring peptide analogue, MART-1. However, the modest increase in response induced by the GLA-SE vaccine, particularly in the context of the small sample size of this study, does not allow us to conclude that GLA-SE is significantly superior as an adjuvant compared with SE alone, which was sufficient to induce CTL responses to peptide in a majority of patients. This limited efficacy suggests that additional vaccine strategies, such as the combination of multiple adjuvants, or the 
incorporation of a helper peptide, may be required to achieve immunogenicity superior to what is possible using SE alone [21,22].

Interestingly, differential induction of cytokines was noted between the two schedules during the first cycle of vaccination. Vaccination with $S E$ was associated with a greater relative increase in pro-inflammatory cytokines such as IL-1 $\beta$, IL-1RA and IL-6 compared with the GLA-SE group. This contrasts with previous in vitro studies, where immune cells stimulated with GLA-SE produced greater levels of pro-inflammatory cytokines than those activated with SE [23]. In both schedules, progressive increases in MCP-1, EGF, TGF- $\alpha$ and sCD40L were observed. Of these factors, $\mathrm{sCD} 40 \mathrm{~L}$ in particular has been observed to enhance CTL responses in a previous melanoma vaccine study that also targeted MART-1, suggesting that increased SCD40L levels may correlate with vaccine response [24]. Our group has also observed consistent increases in patient sCD40L levels in a past MART-1a peptide vaccine trial [25].

Prior studies indicate that $T_{H} 1$ cells play a key role in the development of antitumor CTL responses, either through the secretion of cytokines or the licensing of dendritic cells [26]. In contrast, $\mathrm{T}_{\mathrm{H}} 2$ cells are widely viewed as dispensable or even counter-productive in the development of antitumor immunity [27]. In our studies, both schedules exhibited a progressive increase in the ratios of $\mathrm{T}_{\mathrm{H}} 1$ cells to $\mathrm{T}_{\mathrm{H}} 2$ cells throughout the course of vaccine administration, which suggests that increase in this ratio may correlate with development of an immune response to vaccination.

Of note, patients treated on Schedule 1 demonstrated a greater rate of relapse than patients on Schedule 2. Among the GLA-SE group, we were surprised to find that patients who later developed recurrence had higher levels of several types of cytokines after vaccine treatment relative to patients who remained in remission. Previously, it has been shown that patients with Stage IV melanoma have higher plasma levels of multiple cytokines relative to patients with earlier stages of melanoma [28]. However, it is not clear whether an exaggerated cytokine response to vaccine treatment creates an immune milieu that fosters melanoma recurrence, or whether the cytokine responses are simply a byproduct of a higher residual tumor burden. In both Schedule 1 and 2, increased risk of recurrence was concordant with advanced disease stage, suggesting that patients with advanced melanoma experienced a greater rate of recurrence regardless of vaccine treatment.

\section{Conclusion}

In summary, this Phase I study demonstrates the feasibility of MART-1a-directed approaches for the treatment of advanced melanoma. Immunization with both GLA-SE and SE augmented the frequency of T cells directed against tumor-relevant antigens, with differential cytokine signatures and increases in the ratio of $\mathrm{T}_{\mathrm{H}} 1 / \mathrm{T}_{\mathrm{H}} 2$ cells noted between schedules. Peptide vaccination was well-tolerated and associated with no grade 3 or higher adverse events related to treatment. Response rates were similar between groups vaccinated with GLA-SE and SE alone, suggesting that future studies should employ additional agents such as adjuvant checkpoint inhibitors or novel immunomodulators to augment the effect of vaccine monotherapy. Although this preliminary study cannot be used to predict clinical efficacy, the immune signatures and cellular responses exhibited in enrollees provide initiative for further investigation into combination vaccine formulations that may 1 day improve outcomes for patients with melanoma.

\section{Future perspective}

Going forward, there are three key questions in the field of peptide vaccine immunotherapy that must be answered to facilitate the development of an efficacious melanoma vaccine. The first regards antigen choice, with current debate centered around the benefits and limitations of tumor-associated antigens (TAAs) versus tumor-specific neoantigens. Here, we have demonstrated that MART-1a peptide, which is derived from a TAA, can be easily manufactured and safely administered to patients. However, intrinsic tolerance mechanisms may limit the efficacy of TAA-based vaccines even if they are capable of inducing antigen-specific T cells. In contrast, neoantigenbased vaccines are able to circumvent tolerance and induce meaningful responses, as demonstrated by multiple groups $[29,30]$. The key limitation of this approach is antigen discovery, which relies on cumbersome sequencing or liquid chromatography-mass spectrometry pipelines to identify candidate neoantigens. Moreover, many proposed neoantigens possess sequences that cannot be synthesized using current chemistry. In the near future, workflows to streamline neoantigen identification and synthesis may help boost the therapeutic application of cancer vaccines. The second key question involves adjuvant mixture, which we have explored in this study using GLA in combination with SE. This format of mixing a TLR agonist with an oil-in-water emulsion has proven popular in human clinical trials due to its ease of preparation and immunogenicity [31,32]. However, additional approaches may be required not 
only to induce antigen-specific immune responses, but also for enhancement of trafficking of immune cells to and from the site of vaccine administration, such as the addition of toxoids or depot agents [33]. Finally, optimizing the co-administration of peptide vaccines with additional adjuvant therapies, such as costimulatory agents or checkpoint inhibitors, will be key to ensure that vaccine-induced $\mathrm{T}$ cells are not subsequently suppressed by residual tumor cells. Overcoming these three challenges may pave the way for future vaccine-based eradication of melanoma.

\section{Summary points}

- Glucopyranosyl lipid A (GLA) in stable oil-in-water emulsion (SE) is a novel tumor vaccine adjuvant with the potential to augment the antitumor immune response in patients with resected melanoma.

- Study participants (NCT02320305) received up to three cycles of a heteroclitic melanoma antigen peptide (MART-1a) with GLA-SE or SE only, followed by measurement of antigen-specific immune responses, plasma cytokine changes and shifts in key immune cell populations.

- A total of $70.0 \%$ of patients receiving GLA-SE and $63.6 \%$ of patients receiving SE developed immune responses to MART-1a, which translated to immune response rates against wild-type MART-1 of 40.0 and $54.5 \%$, respectively.

- Indicators of immune response, such as upregulation of pro-inflammatory cytokines and an increase of the relative frequency of $\mathrm{T}_{\mathrm{H}} 1$ cells, were observed to a greater degree in the SE group.

- Vaccination on both schedules was associated with minimal adverse effects, with no dose-limiting toxicities.

- Due to the similarity in rates of vaccine response between both groups, additional strategies are warranted to enhance the clinical efficacy of peptide vaccines for melanoma immunotherapy in the future.

\section{Supplementary data}

To view the supplementary data that accompany this paper please visit the journal website at: www.futuremedicine.com/doi/sup $\mathrm{pl} / 10.2217 / \mathrm{imt}-2020-0085$

\section{Author contributions}

All authors contributed to this work and have read and approved the final manuscript. EP Grewal was responsible for data analysis and drafting and revision of the manuscript. CL Erskine and WK Nevala were responsible for generating laboratory data. JB Allred and was responsible for study design, data analysis and revision of the manuscript. CA Strand was responsible for data analysis. LA Kottschade was responsible for study design and recording clinical data. RR McWilliams and RS Dronca were responsible for recording clinical data. AJ Yakovich was responsible for study design and revision of the manuscript. SN Markovic was responsible for study conception and design, recording clinical data and revision of the manuscript. MS Block was responsible for study conception and design, recording clinical data, data analysis and revision of the manuscript.

\section{Acknowledgments}

The authors wish to acknowledge RK Bradshaw and HJ Turner for coordination of clinical trial activities, LM Flickinger for protocol development assistance, JL Lee for nursing assistance and M Schardt for administrative support.

\section{Financial \& competing interests disclosure}

The authors received funding for this manuscript from the Schulze Foundation. MS Block has received institutional research support from Immune Design, Inc. and Merck, Inc. AJ Yakovich is an employee of Merck, Inc. and Immune Design, Inc. The authors have no other relevant affiliations or financial involvement with any organization or entity with a financial interest in or financial conflict with the subject matter or materials discussed in the manuscript apart from those disclosed.

No writing assistance was utilized in the production of this manuscript.

\section{Ethical conduct of research}

The protocol was approved by the Mayo Clinic Institutional Review Board and all patients underwent written informed consent prior to registration and study participation. The research was conducted according to the principles of the Declaration of Helsinki.

\section{Data sharing statement}

The authors certify that this manuscript reports original clinical trial data and was registered at ClinicalTrials.gov as NCT02320305. No individual participant data that underlies the results reported in the article will be available and nor will information regarding the study protocol or statistical analysis, beyond what is reported in the text. 
Open access

This work is licensed under the Attribution-NonCommercial-NoDerivatives 4.0 Unported License. To view a copy of this license, visit http://creativecommons.org/licenses/by-nc-nd/4.0/

\section{References}

Papers of special note have been highlighted as: $\bullet$ of interest; $\bullet \bullet$ of considerable interest

1. Sosman JA, Moon J, Tuthill RJ et al. A Phase II trial of complete resection for stage IV melanoma: results of Southwest Oncology Group Clinical Trial S9430. Cancer 117(20), 4740-06 (2011).

2. Weber J, Mandala M, Del Vecchio M et al. Adjuvant nivolumab versus ipilimumab in resected stage III or IV melanoma. N. Engl. J. Med. 377, 1824-1835 (2017).

3. Van den Eynde B, Hainaut P, Hérin M et al. Presence on a human melanoma of multiple antigens recognized by autologous CTL. Int. J. Cancer 44(4), 634-640 (1989).

4. Weber JS, Hua FL, Spears L et al. A Phase I trial of an HLA-A1 restricted MAGE-3 epitope peptide with incomplete Freund's adjuvant in patients with resected high-risk melanoma. J. Immunother. 22(5), 431-440 (1999).

5. Kenter GG, Welters MJ, Valentijn AR et al. Vaccination against HPV-16 oncoproteins for vulvar intraepithelial neoplasia. N. Engl. J. Med. 361(19), 1838-1847 (2009).

6. Kawakami Y, Eliyahu S, Delgado $\mathrm{CH}$ et al. Cloning of the gene coding for a shared human melanoma antigen recognized by autologous T cells infiltrating into tumor. Proc. Natl Acad. Sci. USA 91, 3515-3519 (1994).

-• Here, MART-1 was first validated as a tumor-relevant antigen for T-cell targeting.

7. Block MS, Suman VJ, Nevala WK et al. Pilot study of granulocyte-macrophage colony-stimulating factor and interleukin-2 as immune adjuvants for a melanoma peptide vaccine. Melanoma Res. 21, 438-445 (2011).

8. Slingluff CL Jr, Petroni GR, Yamshchikov GV et al. Immunologic and clinical outcomes of vaccination with a multiepitope melanoma peptide vaccine plus low-dose interleukin-2 administered either concurrently or on a delayed schedule. J. Clin. Oncol. 22(22), 4474-4485 (2004).

9. Khong H, Overwijk WW. Adjuvants for peptide-based cancer vaccines. J. Immunother. Cancer. 4, 56 (2016).

10. Slingluff CL Jr. The present and future of peptide vaccines for cancer: single or multiple, long or short, alone or in combination? Cancer J. 17(5), 343-350 (2011).

11. Maurer DM, Butterfield LH, Vujanovic L. Melanoma vaccines: clinical status and immune endpoints. Melanoma Res. 29(2), 109-118 (2019).

- An excellent review of current advances in vaccine therapy for melanoma.

12. Schneider LP, Schoonderwoerd AJ, Moutaftsi M et al. Intradermally administered TLR4 agonist GLA-SE enhances the capacity of human skin DCs to activate T cells and promotes emigration of Langerhans cells. Vaccine 30(28), 4216-4224 (2012).

•. The first study demonstrating the immunogenicity of glucopyranosyl lipid A-stable oil-in-water emulsion in the context of cutaneous immunity.

13. Coler RN, Day TA, Ellis R et al. The TLR-4 agonist adjuvant, GLA-SE, improves magnitude and quality of immune responses elicited by the ID93 tuberculosis vaccine: first-in-human trial. NPJ Vaccines 3, 34 (2018).

14. Disis ML. Immune regulation of cancer. J. Clin. Oncol. 28(29), 4531-4538 (2010).

15. Monney L, Sabatos CA, Gaglia JL et al. Th1-specific cell surface protein Tim-3 regulates macrophage activation and severity of an autoimmune disease. Nature 415(6871), 536-541 (2002).

16. Lee CL, Chiu PC, Lam KK et al. Differential actions of glycodelin-A on Th-1 and Th-2 cells: a paracrine mechanism that could produce the Th-2 dominant environment during pregnancy. Hum. Reprod. 26(3), 517-526 (2011).

17. Simms PE, Ellis TM. Utility of flow cytometric detection of CD69 expression as a rapid method for determining poly-and oligoclonal lymphocyte activation. Clin. Diagn. Lab. Immunol. 3(3), 301-304 (1996).

18. Hengel RL, Thaker V, Pavlick MV et al. Cutting edge: 1-selectin (CD62L) expression distinguishes small resting memory CD4+ T cells that preferentially respond to recall antigen. J. Immunol. 170(1), 28-32 (2003).

19. Bezu L, Kepp O, Cerrato G et al. Trial watch: peptide-based vaccines in anticancer therapy. Oncoimmunology 7(12), e1511506 (2018).

20. Duthie MS, Windish HP, Fox C et al. Use of defined TLR ligands as adjuvants within human vaccines. Immunol. Rev. 239(1), 178-196 (2011).

21. Levast B, Awate S, Babiuk L, Mutwiri G, Gerdts V, van Drunen Littel-van den Hurk S. Vaccine potentiation by combination adjuvants. Vaccines (Basel) 2(2), 297-322 (2014).

22. Reed CM, Cresce ND, Mauldin IS, Slingluff CL Jr, Olson WC. Vaccination with melanoma helper peptides induces antibody responses associated with improved overall survival. Clin. Cancer. Res. 21(17), 3879-3887 (2015). 
23. Coler RN, Bertholet $\mathrm{S}$, Moutaftsi $\mathrm{M}$ et al. Development and characterization of synthetic glucopyranosyl lipid adjuvant system as a vaccine adjuvant. PLoS ONE 6(1), e16333 (2011).

24. Onaitis MW, Kalady MF, Emani $\mathrm{S}$ et al. CD40 ligand is essential for generation of specific cytotoxic T cell responses in RNA-pulsed dendritic cell immunotherapy. Surgery 134(2), 300-305 (2003).

25. Block MS, Nevala WK, Pang YP et al. A pilot clinical trial testing topical resiquimod and a xenopeptide as immune adjuvants for a melanoma vaccine targeting MART-1. Melanoma Res. 29(4), 420-427 (2019).

26. Smith CM, Wilson NS, Waithman J et al. Cognate CD4(+) T cell licensing of dendritic cells in CD8(+) T cell immunity. Nat. Immunol. 5(11), 1143-1148 (2004).

27. Knutson KL, Disis ML. Tumor antigen-specific T helper cells in cancer immunity and immunotherapy. Cancer Immunol. Immunother. 54(8), 721-728 (2005).

28. Nevala WK, Vachon CM, Leontovich AA et al. Evidence of systemic Th2-driven chronic inflammation in patients with metastatic melanoma. Clin. Cancer Res. 15(6), 1931-1939 (2009).

- Provides insights into cytokine signatures that may be observed in melanoma patients.

29. Ott PA, Hu Z, Keskin DB et al. An immunogenic personal neoantigen vaccine for patients with melanoma. Nature 547(7662), 217-221 (2017).

30. Hilf N, Kuttruff-Coqui S, Frenzel K et al. Actively personalized vaccination trial for newly diagnosed glioblastoma. Nature 565(7738), 240-245 (2019).

31. Sabado RL, Pavlick A, Gnjatic S et al. Resiquimod as an immunologic adjuvant for NY-ESO-1 protein vaccination in patients with high-risk melanoma. Cancer Immunol. Res. 3(3), 278-287 (2015).

32. Bekaii-Saab T, Wesolowski R, Ahn DH et al. Phase I Immunotherapy trial with two chimeric HER-2 B-cell peptide vaccines emulsified in montanide ISA 720VG and nor-MDP adjuvant in patients with advanced solid tumors. Clin. Cancer Res. 25(12), 3495-3507 (2019).

33. Mehta NK, Moynihan KD, Irvine DJ. Engineering new approaches to cancer vaccines. Cancer Immunol. Res. 3(8), 836-843 (2015). 
